\title{
A Novel UWB TEM Horn Antenna with a Microstrip-Type Feed
}

\author{
Youcheng Wang, Guangyou Fang, Hai Su, Yicai Ji, Shengbo Ye, and Xiaojuan Zhang \\ School of Electronic, Electrical and Communication Engineering, University of Chinese Academy of Sciences, Beijing 100190, China
}

Correspondence should be addressed to Youcheng Wang; wangyoucheng11@mails.ucas.ac.cn

Received 20 June 2015; Revised 12 August 2015; Accepted 23 August 2015

Academic Editor: Claudio Gennarelli

Copyright (C) 2015 Youcheng Wang et al. This is an open access article distributed under the Creative Commons Attribution License, which permits unrestricted use, distribution, and reproduction in any medium, provided the original work is properly cited.

\begin{abstract}
A novel ultra-wideband (UWB) TEM horn antenna fed by a microstrip-type transition is designed. The feeding structure is directly realized by a microstrip line with the merit of compact size and UWB. To broaden the low frequency band, an arc surface is added at the end of the flare plates. Numerical simulation is applied to discuss the effect of the geometry construction which contains the microstrip-type transition and the circular arc surface at the end of radiating arm. Return loss, radiation pattern, and gain of the fabricated antennas are measured. The measured results show that the antenna yields a wide bandwidth ranging from $1 \mathrm{GHZ}$ to $10.3 \mathrm{GHz}$ with return loss less than $-10 \mathrm{~dB}$. The antenna also achieves unidirectional radiation patterns with stable antenna gain. These characteristics imply that the compact antenna may be used for detecting buried objects in the wall or UWB communication systems of band like GSM, Wi-Fi, and so forth.
\end{abstract}

\section{Introduction}

TEM horn antenna is commonly used due to its relatively high gain, wideband, and simple structure for various applications $[1,2]$. Typical applications include EMC experiments and ground penetrating radar (GPR), detecting hidden objects in wall and UWB communication [3-7]. To radiate electromagnetic waves, the dimension and impedance of the TEM horn antenna should be properly chosen to achieve wideband characteristics [8]. The impedance variation of the tapered plates can be linear [9], Chebyshev type [3], exponential [6], and elliptic profile [10]. Linearly tapered antennas can be constructed easily compared to a Chebyshev type or exponentially tapered antenna. A Chebyshev type tapered antenna could improve the directivity at a fixed taper length. The exponentially tapered plates can achieve a smoother impedance variation. Meanwhile, a worthy feeding mechanism can broaden the bandwidth to a great extent. Typically the TEM horn antenna is fed by a coaxial line [10]. In order to match the impedance between the horn antenna and the unbalanced coaxial line, a compact microstrip-type transition is needed [6]. In the letter, a novel TEM horn antenna fed by a compact microstrip line with asymmetrical section is proposed. The antenna which is modified on the base of [2] is made up of two specially tapered plates and directly fed with a $50 \mathrm{ohm}$ coaxial cable. However, in this paper, an arc surface is added at the end of the flare plates, and the taper is optimized to minimize the reflection. The width of reflector is varied along with the impedance changing from $50 \mathrm{ohms}$ to $370 \mathrm{ohms}$. Finally, pairs of antenna are manufactured and measured. The measured results show that the proposed antenna has low return loss within a wideband from $1 \mathrm{GHz}$ to $10.3 \mathrm{GHz}$ and moderate gain more than $6 \mathrm{dBi}$ in frequencies higher than $2.5 \mathrm{GHz}$. The experimental results agree well with the simulated results.

\section{Antenna Design and Structure}

Based on the transfer line theory, a modification of DolphChebyshev taper presents that the shortest length of the antenna can be designed with a limited frequency band or the maximum bandwidth can be achieved with a given reflection coefficient [11]. Some TEM horn antennas have been published with excellent frequency characters referred to the theory $[12,13]$. In the paper [14], the main reflections of horn antenna are divided into port reflections, taper reflections, and aperture reflections. The port reflections can 


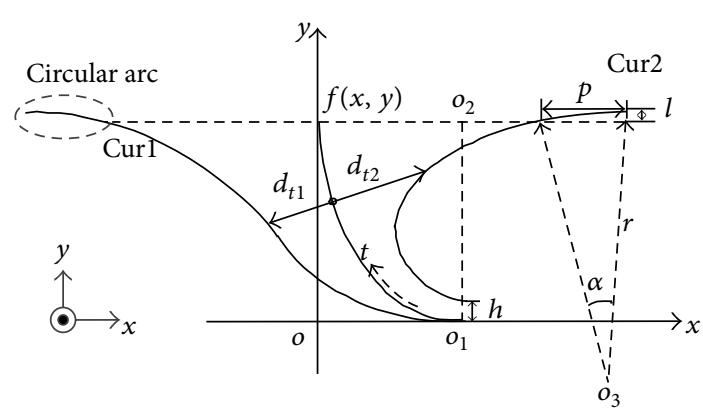

(a)

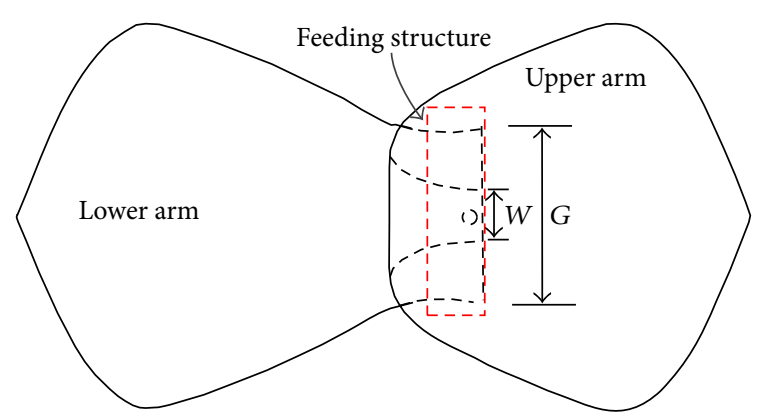

(b)

FIGURE 1: The geometry structure of antenna.

be reduced by transformer matching. The aperture and taper reflections may be determined by the dimension from throat to aperture of horn antenna. In this paper, the taper of proposed antenna is derived from the superellipse function with the power equal to 3 . The varied power could control the form of the superellipse, and the value of 3 is defined for shaping the taper to achieve the smooth current distribution. The geometry of the proposed antenna is illustrated in Figure 1. The structure of the antenna can be divided into two parts including the asymmetrical radiation section and a microstrip-type feeding. The asymmetrical radiation section comprises a pair of tapered metal sheets to accomplish the radiation. The side view and top view of the radiation section are illustrated in Figure 1. A quarter of a cubic hyper elliptic curve with $a=20 \mathrm{~mm}$ and $b=32.25 \mathrm{~mm}$ is chosen as the based reference function $f(x, y)$ corresponding to the expression (1), and the parameter $t$ determines the curved length with relation to the origin $o_{1}$. The center of the cubic hyper elliptic curve is $o_{2}$, whose coordinates are of $20 \mathrm{~mm}$ and $32.25 \mathrm{~mm}$. The curves of the taper are computed through the parametric functions $d_{t 1}$ and $d_{t 2}$ versus the parameter $t$ according to expressions (2) and (3). To broaden the low frequency band, two symmetrical circular arcs with the radius $r=53.845 \mathrm{~mm}$ and the angle $\alpha=42.5$ degree are loaded at the end of the flare-shaping curves with the center $o_{3}$ of $59.72 \mathrm{~mm}$ and $-12.65 \mathrm{~mm}$. The width of horn's cross section varies with the distance between Curl and Cur2 as parallel waveguide or microstrip line $[3,14]$, and the microstrip-type structure is shown as dot line in Figure 1(b). The microstriptype structure with air substrate should be matched to the 50$\mathrm{ohm}$ coaxial cable. According to the impedance calculation of the microstrip line, the initial value of $h$ is $1.75 \mathrm{~mm}, w$ is $7 \mathrm{~mm}$, and $G$ is $35 \mathrm{~mm}$.

The manufactured antenna is shown in Figure 2. The conducting plates are shaped by the copper with thickness of $0.5 \mathrm{~mm}$ to keep light weight. To support and fix the plates of the antenna, polystyrene foam which conforms to the antenna can be used for engineering application. The dimension of the horn antenna is $136 \times 64 \times 40.5 \mathrm{~mm}$. Compared to the regular horn antenna [15], the proposed horn antenna has a much smaller size and good radiation characteristic. The compact size makes it possible to form
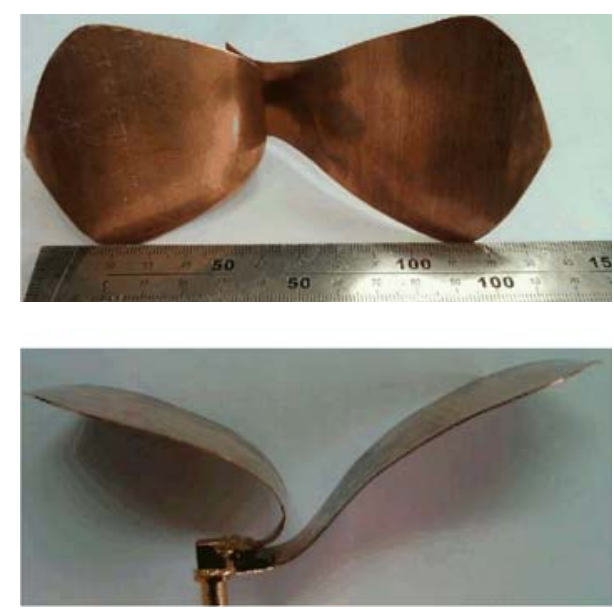

FIGURE 2: Photograph of the manufactured antenna.

antenna array with this antenna to improve the gain for detecting buried targets in the wall or ground:

$$
\begin{aligned}
f(x, y) & =0:\left|\frac{x-a}{a}\right|^{3}+\left|\frac{y-b}{b}\right|^{3}=1 ; \\
\quad 0 \leq x \leq a, 0 \leq y \leq b, & \\
d_{t 1} & =0.44 e^{0.1 t}+0.44, \\
d_{t 2} & =0.4 e^{0.1 t}+0.21 .
\end{aligned}
$$

\section{Parameter Discussion and Experiments}

The frequency band is limited by the dimension of the horn aperture; therefore two symmetrical circular arcs surfaces are loaded at the end of flare plates. Parameters $p$ and $l$ are critical to the circular arc surface. In Figure 3(a), when $p$ varies from $34 \mathrm{~mm}$ to $42 \mathrm{~mm}$ with a increment of $4 \mathrm{~mm}, S_{11}$ varies slightly from 1 to $10.5 \mathrm{GHz}$ and less than $-10 \mathrm{~dB}$. In Figure 3(b), as $l$ varies from $4.3 \mathrm{~mm}$ to $12.3 \mathrm{~mm}$ with a increment of $4 \mathrm{~mm}$, the return loss changes slightly except for the frequency at $2.5 \mathrm{GHz}$ with $l=4.3 \mathrm{~mm}$ and $l=12.3 \mathrm{~mm}$. To validate 


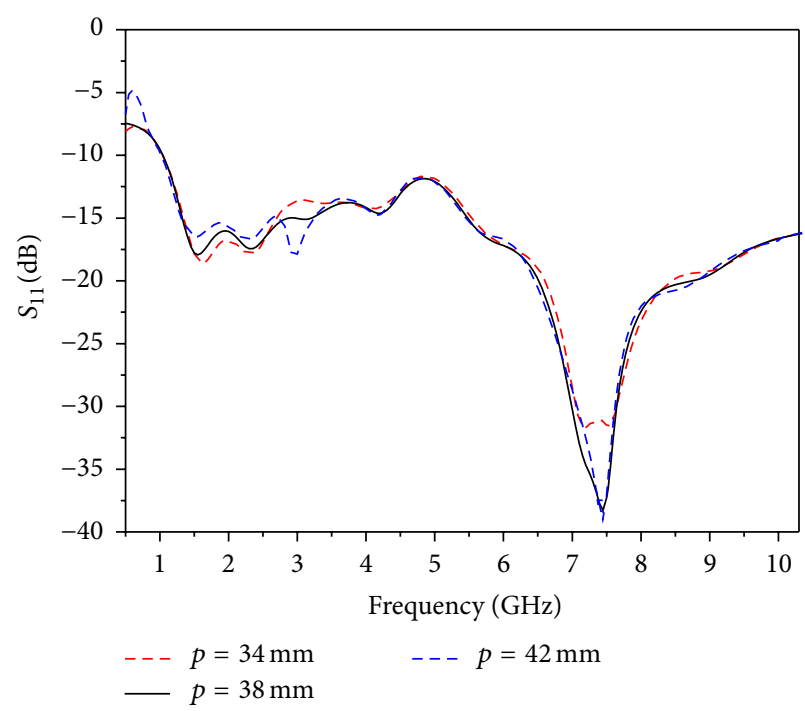

(a)

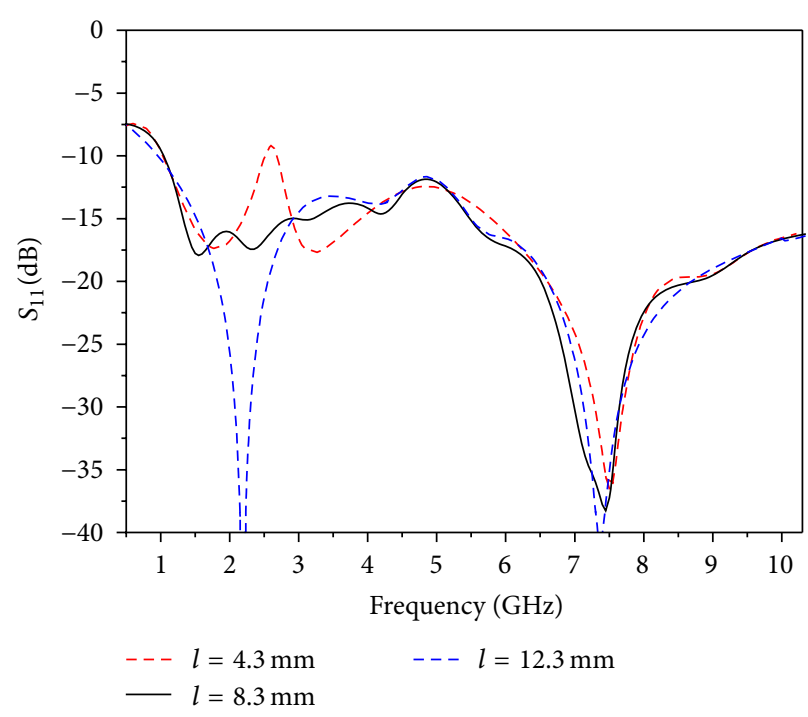

(b)

FIGURE 3: Simulated return loss of proposed antenna at (a) height of the arc and (b) length of the arc.

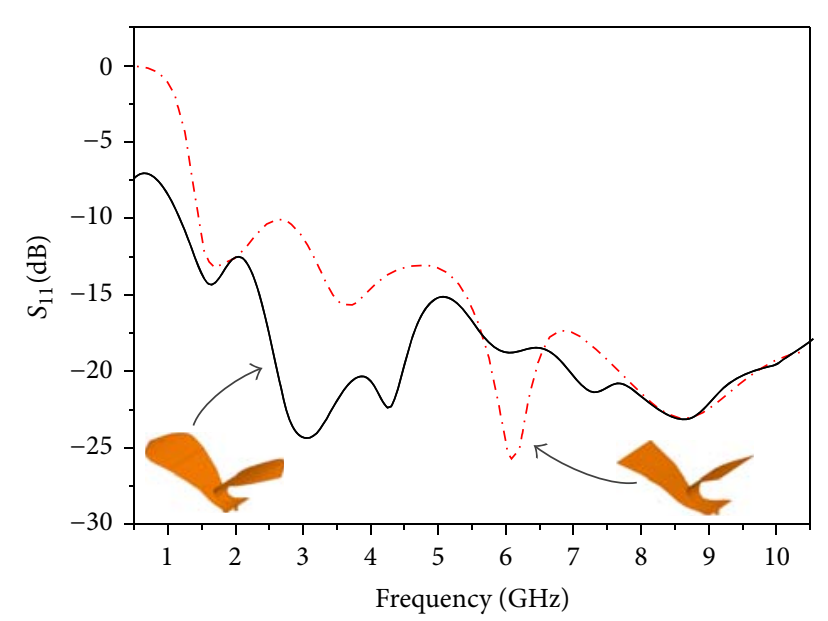

- With arc surface

. . - No arc surface

FIGURE 4: The different return loss between the arc surface loading or not.

the effectiveness of loading circular arc surface, the simulated return loss of antennas with and without arc surface loadings is shown in Figure 4 . The parameter $h$ is defined to $1.5 \mathrm{~mm}$, $p=38 \mathrm{~mm}$, and $l=8.3 \mathrm{~mm}$, which can imply that the low frequency band is mostly affected by the dimension of aperture. The antenna without arc surface loading performs poorly in low frequency band. While the arc surfaces are loaded, the return loss shifts down from $1.45 \mathrm{GHz}$ to $1.1 \mathrm{GHz}$ and smaller than $-10 \mathrm{~dB}$. From $2 \mathrm{GHz}$ to $5 \mathrm{GHz}$, that is, the return loss achieves lower level by loading the arc surface.

The parameters $w$ and $h$ of microstrip-type transition are learnt by simulation. Figures 5 and 6 illustrate the effects of the width $w$ of the microstrip and the thickness $h$ of the air substrate on return loss, respectively. In Figure 5, it is

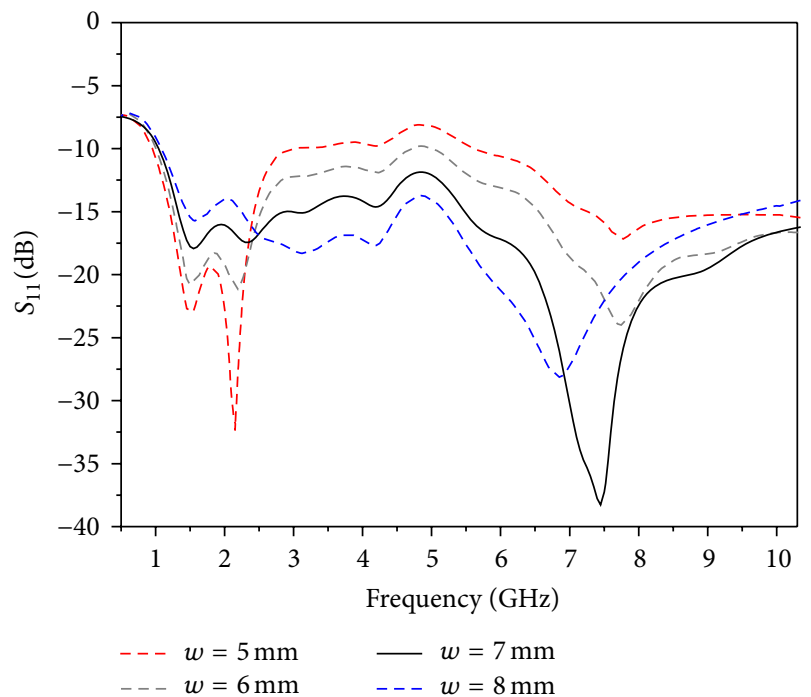

FIGURE 5: Simulated return loss of proposed antenna at width of microstrip.

obvious that the antenna performs better in high frequency band when the width of microstrip $w$ varies from 5 to $8 \mathrm{~mm}$. Here the values of $h, p$, and $l$ are chosen to be $2 \mathrm{~mm}$, $38 \mathrm{~mm}$, and $8.3 \mathrm{~mm}$, respectively. Figure 6 shows that the return loss in low frequency band becomes lower than $-10 \mathrm{~dB}$ when height $h$ varies from $1.0 \mathrm{~mm}$ to $2.5 \mathrm{~mm}$, while in high frequency band it changes slightly. Finally, the geometry of microstrip-type transition is defined with $h$ equal to $1.5 \mathrm{~mm}$ and $w$ equal to $7 \mathrm{~mm}$. The simulated input impedance for the proposed antenna is shown in Figure 7. The real part of impedance is around $50 \mathrm{ohms}$, and the imaginary part is close to zero with small fluctuations in the whole band. It means that the antenna would be matched well with port 


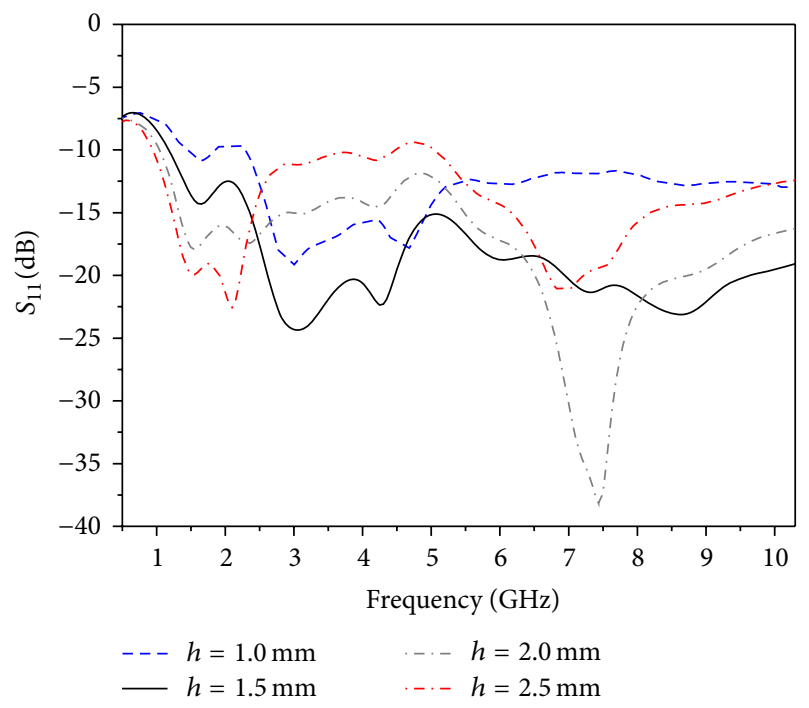

FIGURE 6: Simulated return loss of proposed antenna at height of air substrate.

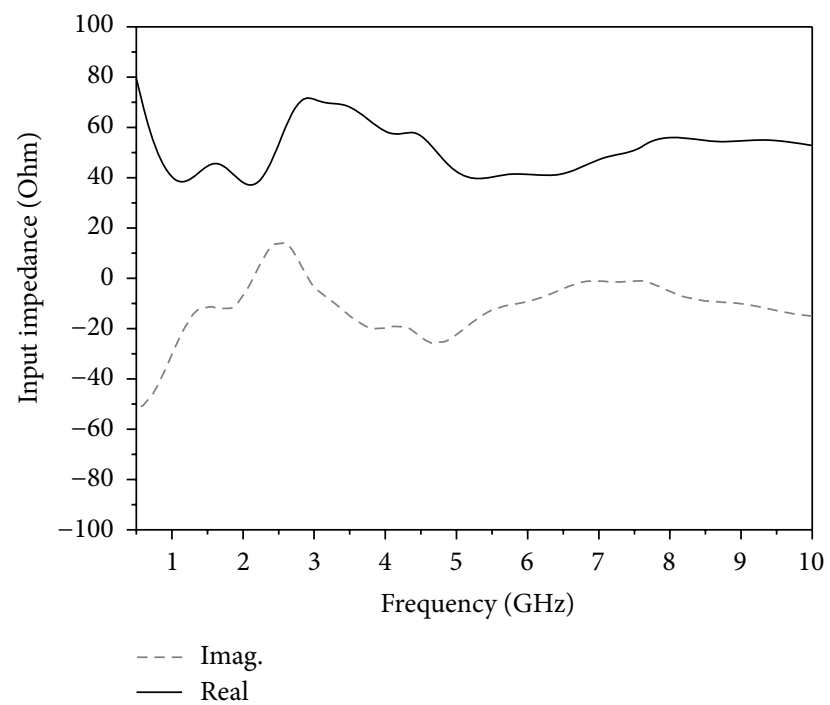

FIGURE 7: Simulated input impedance of proposed antenna.

of RF connector. The constructed antennas are measured by using Agilent network analyzer N5242A with thru-reflect line calibration to exclude the effects of the connectors and two $50-\mathrm{ohm}$ coaxial cables. The measured results agree well with simulation except at the frequency around $10 \mathrm{GHz}$ as shown in Figure 8. This can be ascribed to the fabrication discrepancy of the horn antenna throat which is made in hand. The RF connectors and coaxial cables may also contribute slightly to the discrepancy at high frequencies. The measured $E$-plane and $H$-plane radiation patterns at the frequencies of 1, 4, 7, and $10 \mathrm{GHz}$ are shown in Figure 9. It can be seen that the maximal radiation is along the central axis of the horn aperture in $H$-plane ( $y o z$-plane), while a declination angle of approximately 15 degrees away from the central axis of the aperture is shown in E-plane (xoy-plane). Due to the small size of the antenna, the radiation pattern at $1 \mathrm{GHz}$ is

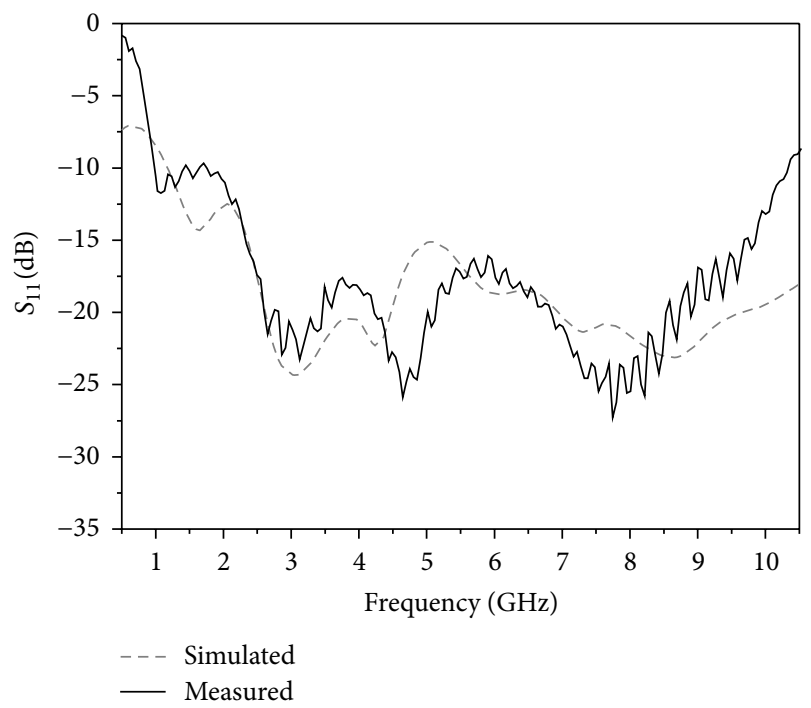

Figure 8: Measured and simulated input return loss of proposed antenna.

similar to a dipole. As the frequency increases, the radiation pattern shows the characteristic of end-fire type antenna. It is observed that the main lobe patterns keep stable, and the half power beam width of the antenna is about 70 degrees. The front-to-back ratio of the antenna is approximately $13 \mathrm{~dB}$. It is observed that some ripples appeared in the radiation pattern as frequency increases, which can be ascribed to the dimension of lower arm. The curved part of the lower arm near feeding transition can be considered to be a reflector, especially at high frequencies. However, it could not be too large in order to balance the size and the directivity of the antenna.

The peak gain of the proposed antenna from $1 \mathrm{GHz}$ to 10.3 GHz is measured using two-uniform-antenna method and shown in Figure 10. The measured and simulated gain curves show a common trend and agree well with each other, the slight differences may be attributed to the limitation of the measurement condition and the manufacturing errors. As shown in Figure 10, the antenna gain is almost constant in the main frequency bandwidth. Antenna gain is higher than $0 \mathrm{dBi}$ at frequencies higher than $1 \mathrm{GHz}$ and remains relatively stable around $6 \mathrm{dBi}$ from $2.5 \mathrm{GHz}$ to $10 \mathrm{GHz}$. The antenna can be used as array element when detecting objects buried in wall or ground [7]. Antenna array could improve the gain which may meet requirements of compensating attenuation.

\section{Conclusion}

A novel UWB TEM horn antenna which is fed by a compact microstrip-type transition and loaded with circular arc surface has been numerically modeled and experimentally investigated. The geometry construction of the microstriptype transition and the circular arc surface which affects the return loss of the antenna are simulated and discussed. The measured and numerical results are in good agreement with each other. The measured results show that the proposed 


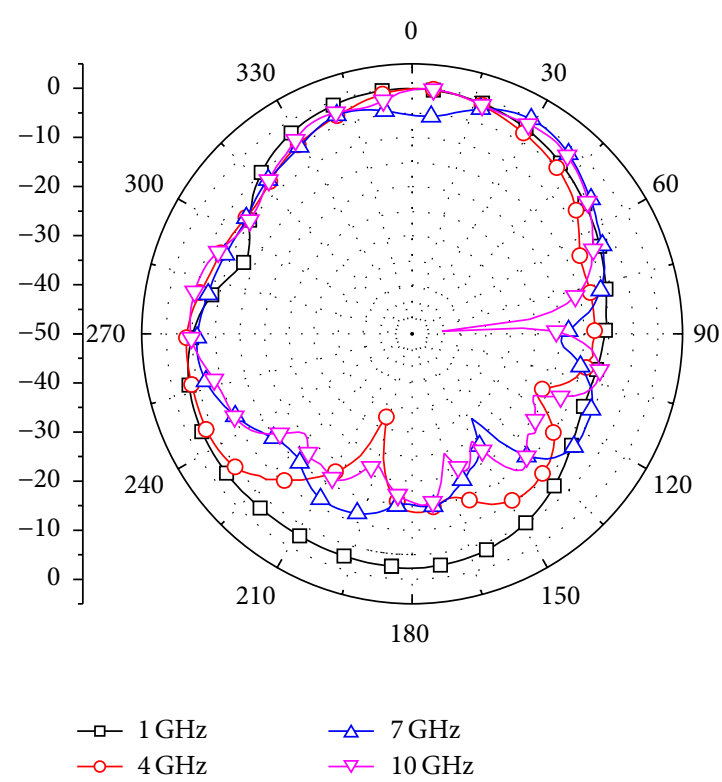

(a)

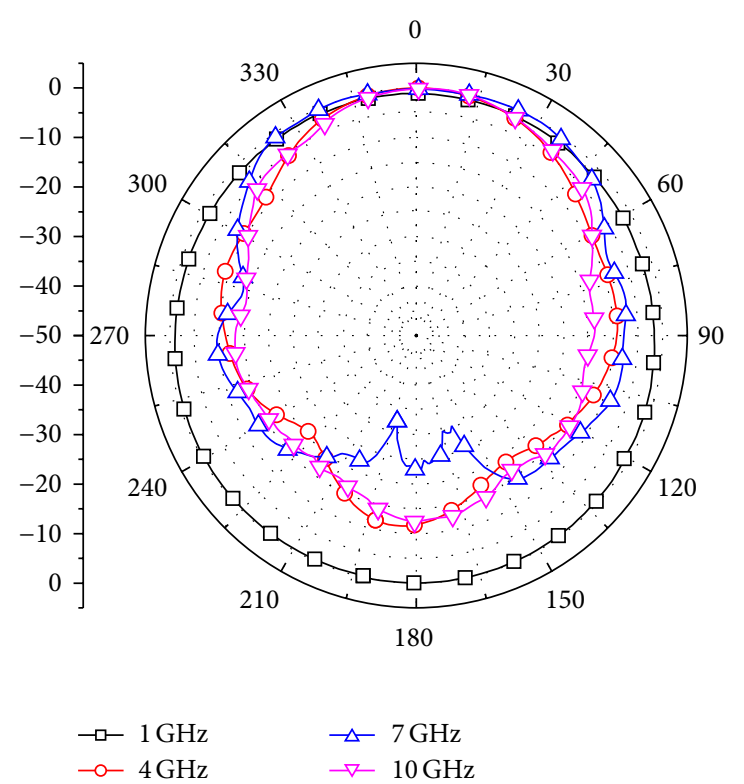

(b)

FIGURE 9: Normalized measured radiation patterns for proposed antenna:(a) $E$-plane and (b) $H$-plane.

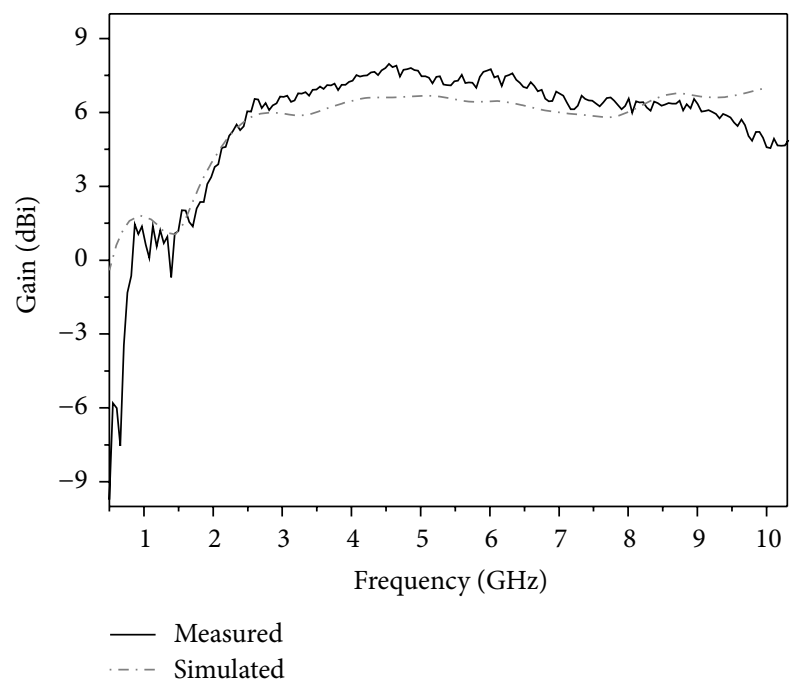

FIGURE 10: Measured and simulated peak gain of antenna against frequency for proposed antenna. The simulated gain is in the $x o y$ plane with the azimuth angle $\varphi$ equal to 105 degree.

antenna can provide good end-fire radiation patterns and a broadband impedance bandwidth. The gain of the antenna is larger than $6 \mathrm{dBi}$ in almost entire frequency band. These results indicate that the proposed antenna is expected to be a good candidate for some UWB radar systems or local wireless communication owing to its advantages of low cost, light weight, and compact size. To avoid the interference with other major wireless devices, a notch band may be required. However, the horn antenna is not easy to shape notch band. Generally, the unidirectional horn antenna is possible to avoid interference with other wireless communication devices by proper distribution. Radome could also be used to avoid interference. The application issue will be the next important theme.

\section{Conflict of Interests}

The authors declare that there is no conflict of interests regarding the publication of this paper.

\section{Acknowledgments}

This work has been supported by the National Basic Research Program of China ("863" Program) of Grant 2012AA061403 and Technical Program of Beijing Transportation Industry under no. of Y4H33A0154.

\section{References}

[1] A. Mehrdadian and K. Forooraghi, "Design and fabrication of a novel ultrawideband combined antenna," IEEE Antennas and Wireless Propagation Letters, vol. 13, pp. 95-98, 2014.

[2] A. O. Boryssenko and D. L. Sostanovsky, "A new two-flareshaped UWB antenna element," in Proceedings of the 2005 Antenna Applications Symposium, pp. 451-471, September 2005.

[3] S. B. Bassam and J. A. Rashed-Mohassel, "A chebyshev tapered TEM horn antenna," PIERS Online, vol. 2, no. 6, pp. 706-709, 2006.

[4] M. İlarslan, A. S. Türk, S. Demirel, M. E. Aydemir, and A. K. Keskin, "A compact vivaldi shaped partially dielectric loaded TEM horn antenna for UWB communication," International Journal of Antennas and Propagation, vol. 2014, Article ID 847169, 6 pages, 2014.

[5] M. N. Shakib, M. Moghavvemi, and W. N. L. Mahadi, "Design of a compact tuning fork-shaped notched ultrawideband antenna 
for wireless communication application," The Scientific World Journal, vol. 2014, Article ID 874241, 8 pages, 2014.

[6] H. Choi and S. Lee, "Design of an exponentially-tapered TEM horn antenna for the wide broadband communication," Microwave and Optical Technology Letters, vol. 40, no. 6, pp. 531534, 2004.

[7] A. Godard, L. Desrumaux, V. Bertrand et al., "A transient UWB antenna array used with complex impedance surfaces," International Journal of Antennas and Propagation, vol. 2010, Article ID 243145, 8 pages, 2010.

[8] K. Chung, S. Pyun, and J. Choi, "Design of an ultrawideband TEM horn antenna with a microstrip-type balun," IEEE Transactions on Antennas and Propagation, vol. 53, no. 10, pp. 3410-3413, 2005.

[9] K. Moussakhani, R. K. Amineh, and N. K. Nikolova, "Highefficiency TEM horn antenna for ultra-wide band microwave tissue imaging," in Proceedings of the IEEE International Symposium on Antennas and Propagation (APSURSI '11), pp. 127-130, July 2011.

[10] J. A. G. Malherbe and N. Barnes, "TEM horn antenna with an elliptic profile," Microwave and Optical Technology Letters, vol. 49, no. 7, pp. 1548-1551, 2007.

[11] R. P. Hecken, "A near-optimum matching section without discontinuities," IEEE Transactions on Microwave Theory and Techniques, vol. 20, no. 11, pp. 734-739, 1972.

[12] E. A. Theodoru, M. R. Gorman, P. R. Rigg, and F. N. Kong, "Broadband pulse-optimised antenna," IEE Proceedings $\mathrm{H}$ : Microwaves, Optics and Antennas, vol. 128, no. 3, pp. 124-130, 1981.

[13] Y. Huang, M. Nakhkash, and J. T. Zhang, "A dielectric material loaded TEM horn antenna," in Proceedings of the 12th International Conference on Antennas and Propagation (ICAP '03), vol. 2, pp. 489-492, IET, March-April 2003.

[14] A. E.-C. Tan, K. Jhamb, and K. Rambabu, "Design of transverse electromagnetic horn for concrete penetrating ultrawideband radar," IEEE Transactions on Antennas and Propagation, vol. 60, no. 4, pp. 1736-1743, 2012.

[15] D. M. Pozar, Microwave Engineering, John Wiley \& Sons, 2009. 

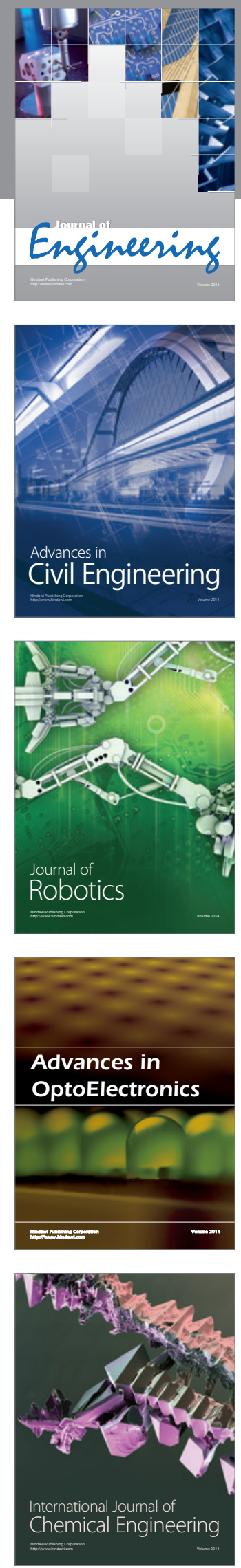

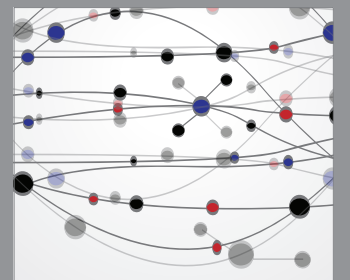

The Scientific World Journal
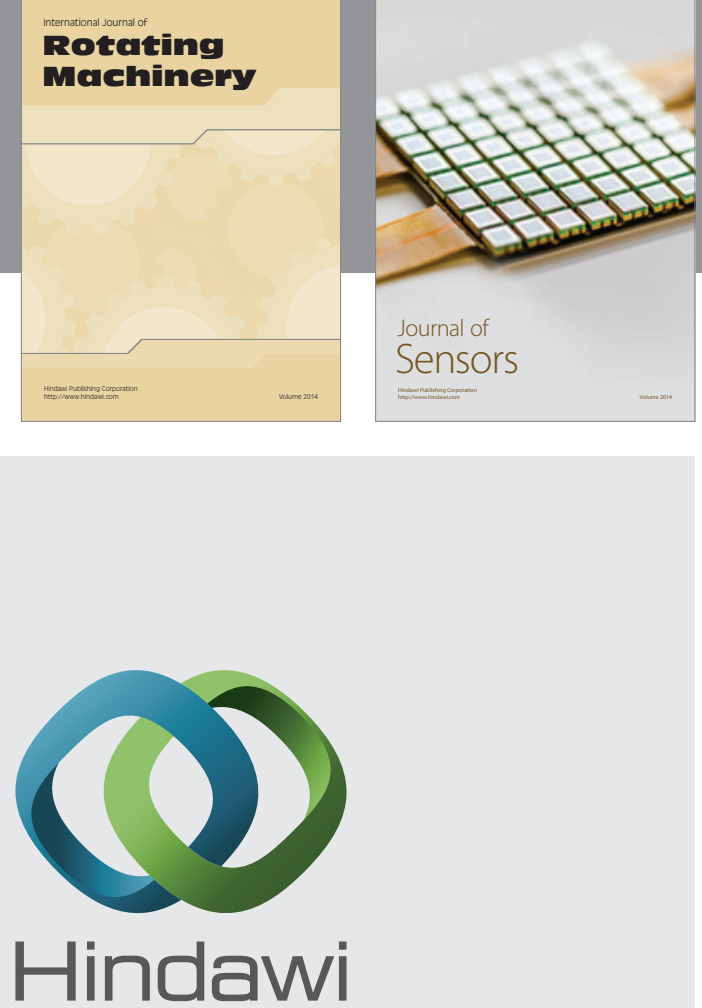

Submit your manuscripts at http://www.hindawi.com
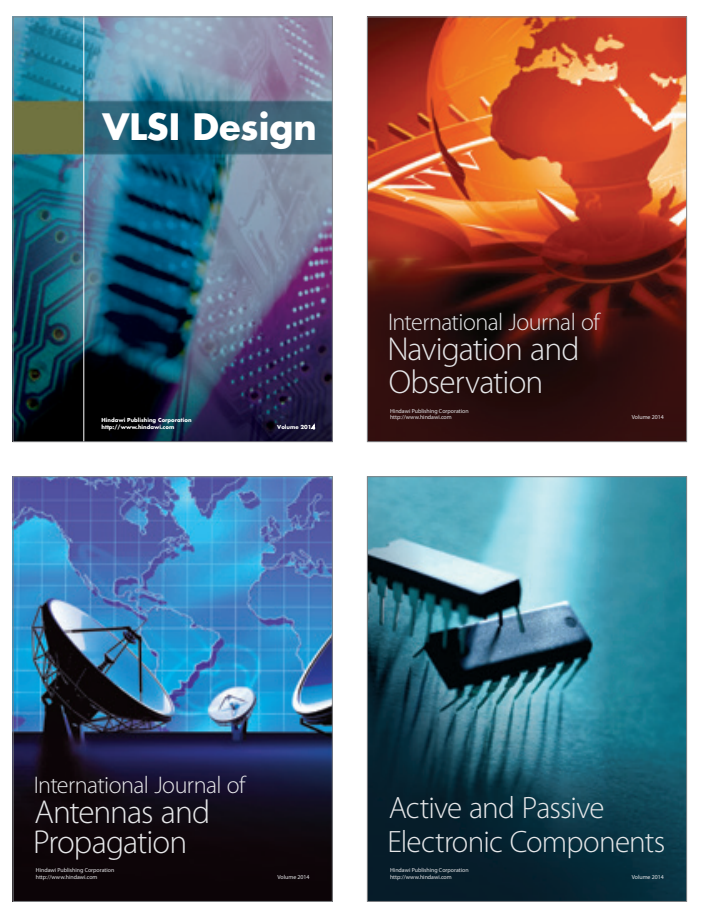
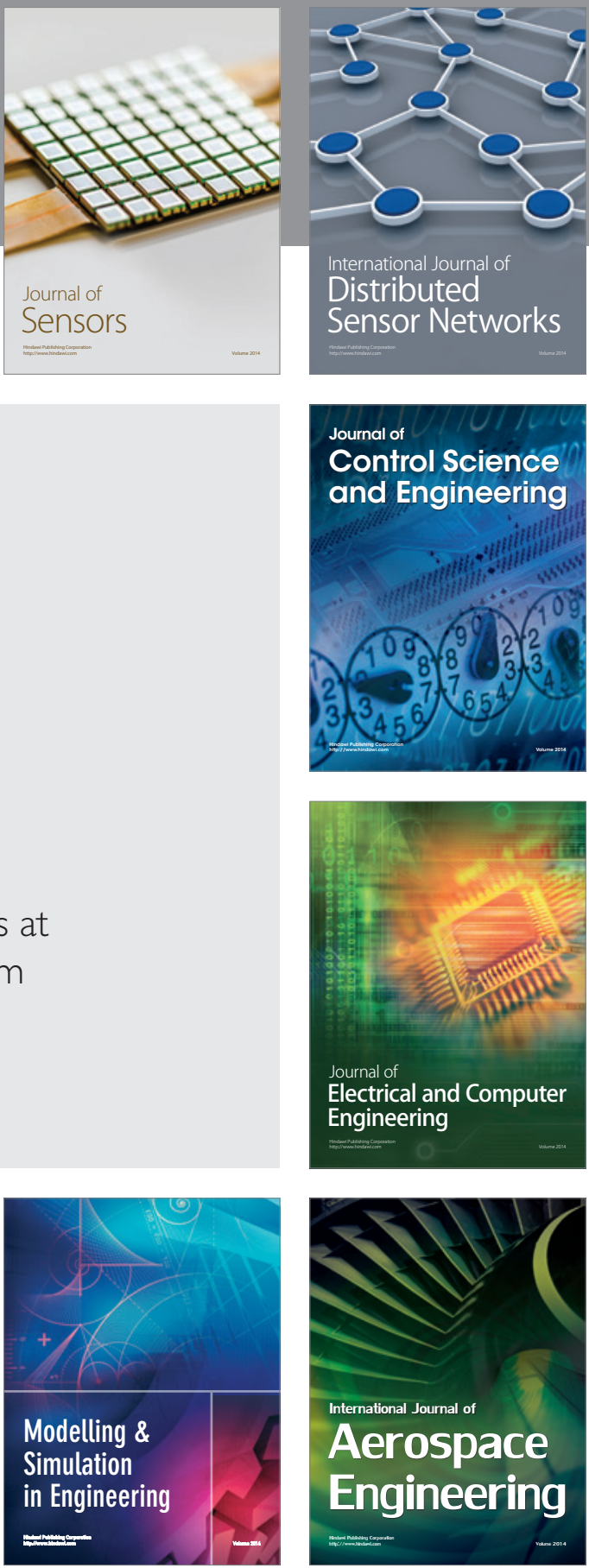

Journal of

Control Science

and Engineering
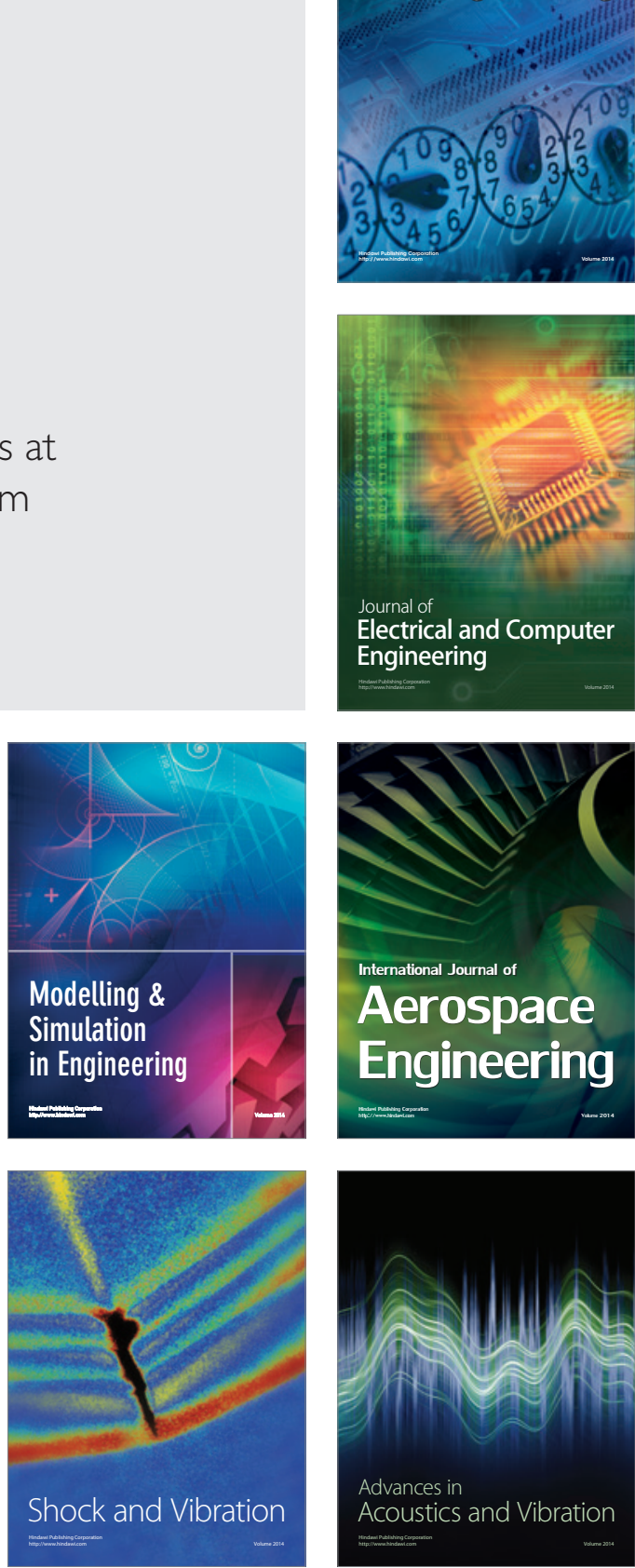